\title{
Decoding the functional role of long noncoding RNAs (IncRNAs) in hypertension progression
}

\author{
Durairaj Sekar ${ }^{1} \cdot$ Deepak Nallaswamy $^{2} \cdot$ Ganesh Lakshmanan $^{3}$
}

Received: 6 February 2020 / Revised: 12 February 2020 / Accepted: 13 February 2020 / Published online: 1 April 2020

(c) The Japanese Society of Hypertension 2020

Hypertension (HTN) or high blood pressure is a predominant noncommunicable disease in the developing and developed world [1-3]. The incidence of HTN has increased rapidly over the years, and the mortality due to HTN was reported to be high in many countries $[4,5]$. Therefore, there is an urgent need for protective measures and treatment options for HTN. Many biomarkers are available to predict or diagnose disease progression, but none of them have been proven to be effective against disease progression. Therapeutic strategies are available for short-term treatment but not for a cure or for the effective management of complications caused by HTN, and endothelial dysfunction is believed to play a major role in the pathogenesis of HTN progression. Long noncoding RNAs (lncRNAs) are a kind of noncoding RNA that are more than 200 nucleotides in length. It has been suggested that lncRNAs are implicated in various cellular processes, including gene expression and epigenetic modifications [6]. Figure 1 depicts the various roles of lncRNAs in cells. Many articles suggest that elevated blood pressure is associated with IncRNAs [7], and lncRNAs may be considered a useful disease biomarker since their expression is observed in circulation.

The functional and biological roles of lncRNAs are not yet fully understood, and the number of articles related to lncRNAs in HTN is sparse. Recently, Jiang and Ning [8] published a review article in HTN Research summarizing

Durairaj Sekar

duraimku@gmail.com

1 Dental Research Cell (DRC-BRULAC), Saveetha Dental College and Hospital, Saveetha Institute of Medical and Technical Science (SIMATS), Saveetha University, Chennai 600077, India

2 Department of Prosthodontics, Saveetha Dental College and Hospital, Saveetha Institute of Medical and Technical Science (SIMATS), Saveetha University, Chennai 600077, India

3 Department of Anatomy, Saveetha Dental College and Hospital, Saveetha Institute of Medical and Technical Science (SIMATS), Saveetha University, Chennai 600077, India the significant role of IncRNAs in HTN progression. Interestingly, the authors tried to link IncRNAs with endothelial cell, vascular smooth muscle cell, renal cell, and cardiac cell pathologies. Like microRNAs, IncRNAs have been widely observed in the blood of HTN patients, suggesting that they may be a potential circulating biomarker for HTN; however, more clarifications and functional studies on lncRNAs are needed. In addition, it has been reported that preeclampsia or pregnancy-induced HTN is linked with lncRNAs [9]. The authors also delineated the critical role of IncRNAs in preeclampsia, but the number of articles related to this study subject is very limited.

The authors of the review paper concluded that IncRNA dysregulation contributes to the pathogenesis of HTN by directly acting on vascular cells and indirectly affecting other organs. Moreover, it is also understandable that we need more future functional studies on IncRNAs with respect to HTN and the mechanism underlying their role in disease progression. Unlike other noncoding RNAs, lncRNAs have their own mechanism of action in cells since they interact with other noncoding RNAs, such as miRNAs, to induce disease progression. Researchers have not yet completely decoded the function of IncRNAs in HTN

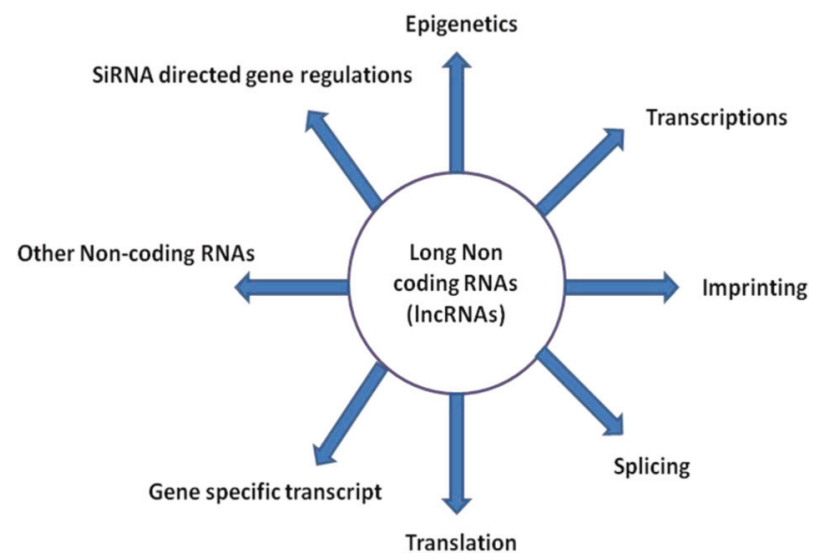

Fig. 1 Depicts the various roles of long noncoding RNAs (lncRNAs) in the cells 
progression since more funding and high-throughput technology are needed. Understanding vascular endothelium dysregulation by noncoding RNAs in HTN may open a new gateway for therapeutic options for the treatment of HTN.

\section{Compliance with ethical standards}

Conflict of interest The authors declare that they have no conflicts of interest.

Publisher's note Springer Nature remains neutral with regard to jurisdictional claims in published maps and institutional affiliations.

\section{References}

1. Sekar D. Circular RNA: a new biomarker for different types of hypertension. Hypertens Res. 2019;42:1824-5.

2. Krishnan R, Mani P, Sivakumar P, Gopinath V, Sekar D. Expression and methylation of circulating microRNA-510 in essential hypertension. Hypertens Res. 2017;40:361-3.
3. Johnson J, Lakshmanan G, M B, R M V, Kalimuthu K, Sekar D. Computational identification of MiRNA-7110 from pulmonary arterial hypertension (PAH) ESTs: a new microRNA that links diabetes and PAH. Hypertens Res. 2020;43:360-2. https://doi.org/ 10.1038/s41440-019-0369-5.

4. Sekar D. Comment on the potential role of microRNAs in hypertension. J Hum Hypertens. 2018;32:639-40.

5. Sekar D, Lakshmanan G, Mani P, Biruntha M. Methylationdependent circulating microRNA 510 in preeclampsia patients. Hypertens Res. 2019;42:1647-8.

6. Jusic A, Devaux Y, EU-CardioRNA COST Action (CA17129). Noncoding RNAs in hypertension. Hypertension. 2019;74:477-92. https://doi.org/10.1161/HYPERTENSIONAHA.119.13412.

7. Jin Q, Zhao Z, Zhao Q, Yu X, Yan L, Zhang $Y$, et al. Long noncoding RNAs: emerging roles in pulmonary hypertension. Heart Fail Rev. 2019. https://doi.org/10.1007/s10741-01909866-2.

8. Jiang $\mathrm{X}$, Ning Q. Long noncoding RNAs as novel players in the pathogenesis of hypertension. Hypertens Res. 2020. https://doi.org/ 10.1038/s41440-020-0408-2.

9. Zhang Y, Zou Y, Wang W, Zuo Q, Jiang Z, Sun M, et al. Downregulated long non-coding RNA MEG3 and its effect on promoting apoptosis and suppressing migration of trophoblast cells. J Cell Biochem. 2015;116:542-50. 